\title{
Tank Unit Network Optimization Simulation System Based IP Multicast
}

\author{
Li Yuanzhou, Wang Ziqiang, Chen Manqing \\ Department of Information Engineering Armored Force \\ Engineering Institute \\ Beijing, China \\ e-mail: lyz156@126.com
}

\author{
Jiang Bingshu \\ Logistics department of the general armament \\ department \\ Beijing, China
}

\begin{abstract}
Distributed Interactive Simulation Technology Development of Tank Unit against the system interconnection in the network communications, there has been problems on the network data redundancy. we use the latest IP Multicast technology to analyze and solve the problem of data redundancy.
\end{abstract}

Keywords-IP multicast, distributed interactive simulation, network optimization, LAN

\section{INTRODUCTION}

Military training is a prerequisite for the military victory achieved in practice proved that the application of training simulators has significant advantages, not only receive good training results, but also save a lot of military expenditure to protect the ecological environment, independent of the natural conditions and political climate. The development of simulation training equipment, lack of funds in the defense of a few cases do more with the way the strong soldiers. However, existing tank combat shooting system simulation, when the network traffic during data redundancy problem, we planned to use IP Multicast technology to solve this problem.

\section{TANK FIRE AGAINST THE STRUCTURE OF THE SYSTEM}

\section{A. Hardware System Structure}

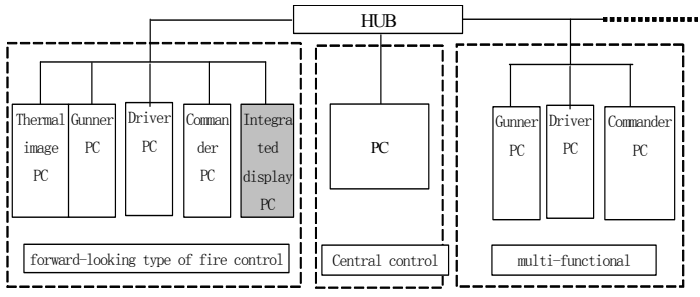

Figure 1. The structure of the simulation node of the " operational effectiveness testing plateform of tank fire control new technique"

"Tank combat simulation training system unit" is composed by the "forward-looking type of fire control simulator simulation platform", the central command station and the "multi-functional simulation platform simulator" .They are interconnected by LAN. "Simulation of Advanced Fire Control Simulator platform" is a simulation node which is composed by five PC machine .They are the bus drivers, gunner, driver, thermal imaging, integrated display. "Multi-functional simulation platform simulator" is composed by drivers, gunner, driver composition. They both are interconnected by the network hub (HUB). Figure 1.

\section{B. Software System Structure}

\section{1) software / protocol level architecture}

In this system, the interconnection between nodes in the network protocol uses TCP / IP protocol, based on the Windows 2000 operating system to $\mathrm{VC}++6.0$ as development environment. The visual simulation software uses OpenGVS. The high-level agreement between the simulation entity HLA uses the distributed interactive simulation agreement. Figure 2:

\begin{tabular}{|c|} 
HLA Protocol \\
\hline $\mathrm{VC}++6.0 /$ OpenGL/OpenGVS \\
\hline Operation system WINDOWS2000/XP \\
\hline Network protocol TCP/IP \\
\hline
\end{tabular}

Figure 2. software system architecture

2) Tank Element combat simulation training system problems

The members of modern tanks are composed of the gunner, the driver and the driving member. To achieve the necessary tank combat simulation to simulate the three crew, the "multi-functional simulation platform simulator" of a tank requires three computer nodes. The traditional mode of operation is that all nodes are connected to the HUB. Figure 3:

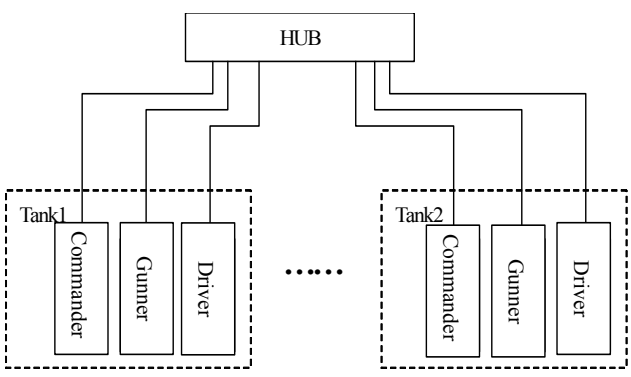

Figure 3. Traditional model

To achieve the above model tank combat simulation is possible, but there are following problems:

(1) complex network data 
The information involved nodes of gunner, monitor and driver are different, such as: the gunner node needs gunner turret angle, angle of the barrel and other information; the driver needs to issue drivers aiming direction information; the driving member needs to publish the location of tanks, direction, speed and other information. So the result is a very complicated data on the network.

(2) personnel operation is difficult

Training in the simulation process, if simulation of five Combat tanks to the other five tanks, there are thirty computers simultaneously send out different information in the network.. The tanks were also made to the internal information network. Exchange of information between the tanks were also made to the network. So if it is necessary of the operator of each simulator to distinguish between what information is sent to their own and what information is of no use, The simulator operator's operating speed will be seriously affected. Moreover, if the operator misjudged the source and destination information, this will lead to disastrous consequences. As a result, the whole simulation process is failure.

(3) the high cost of networking

As in the simulation process, data is very much in the network. So the network hardware configuration is required relatively high against data accumulation problems which will resulti in a simulator crash,

\section{IP Analysis OF Multicast Technology}

\section{A. IP Multicast concepts}

The concept of IP multicast. March was Mentioned for the first time in 1988 in the doctoral thesis of Steve Deering. In 1992, the first test the scope of IETF Internet multicast audio conference was hold, when 20 outlets can hear the voice of the meeting. IP Multicast is a need to add more intelligence can provide a service.

LAN multicast is implemented in hardware. When a PC on the Ethernet receives a frame, the PC-card hardware can determine whether the frame's destination address is one of the following three addresses:

(1) The author card's hardware address (unicast).

(2) the destination address of all 1(broadcast).

(3) the group address which the lowest level of the first byte is 1 and the site has been added to the group (multicast).

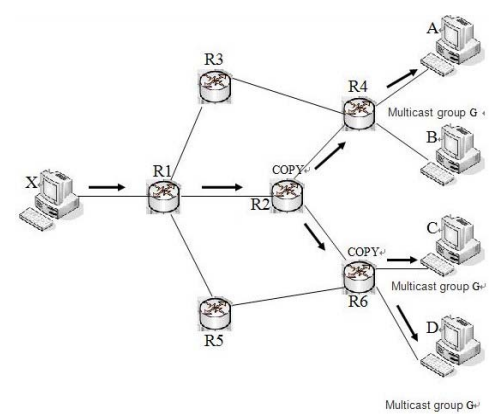

Figure 4. shows multicast
To accept the frame if it is, otherwise to discard it. This may not be a waste of the host processor and memory resources. All the cards should be able to identify at least the first two frames, which are unicast and broadcast address. Some cards can be programmed methods to identify the multicast address. When the operating system starts, it will initialize the card so that the card will identify some of the multicast address.

The Internet to multiple destination station can send the same packet in two ways. One way is to use a single broadcast, in which an end station is sent a packet with many times. Another method is to use multicast. Figure 4.

In figure 4 , the three hosts $\mathrm{A}, \mathrm{C}$, and D compose a multicast group G. Host $\mathrm{X}$ is now to a multicast the three host multicast of group $\mathrm{G}$ (host $\mathrm{X}$ may not belong to the multicast group and does not have to know the multicast group in which members are included.) Only when doing a multicast host sends a datagram to the router R2 is only copy, and then to the R6 to reproduce again. This means that multicast packets only when the bifurcation in the transmission path and continue forwarding packets copied. If it is not multicast, the source sends the outset, standing in 3 packets to host A, C, D. The number of multicast group can be a great host, such as the thousands. Therefore, using the multicast protocol can significantly reduce the consumption of network resources. Clearly, the network is to rely on the multicast router to achieve. So these routers must be able to identify some additional multicast software. The router which is able to run the router multicast protocol is known as multicast router. Multicast router can be a single router or an ordinary multicast router which the software can be run on.

\section{B. IP Multicast has the following features:}

(1) Use the multicast group address

Class D IP address support multicast. Class D IP address prefix is 1110. So the address range is 224.0.0.0 to 239.255.255.255. Each class D address signs for each group of hosts. Class D address can be used to mark each host group, a total of 28bit. So you can mark more than 228 multicast group. When a process sends datagram to a class D address, each host of the group will receive the same packet. The progress is "best effort delivery". But some group members may not receive this data reported.

Obviously, the multicast group address is only for $t$ the destination address and not for the source address.

(2)Permanent group address

Here are a few examples of several permanent group addresses which are assigned by the Internet Authority had IANA (Internet Assigned Numbers Authority):

224.0.0.0 Base Address (Reserved)

224.0.0.1 all participants in the online book multicast hosts and routers

224.0.0.2 all participants in the online book multicast router

239.192.0.0 to 239.251.255.255 limit the scope of an organization

239.252.0.0 to 239.255.255.255 range limit in one place 
(3) the dynamic group membership

Members of the host group is dynamic. Temporary group address is used after creating a host group each time. A process may request the host to participate in a particular group, or at any time to exit the group. When a host participate a new group, it will to send packets to all hosts to state its members relationship. The local multicast router receives the packet and forwards this message to the Internet in other multicast routers. When the last process exits a group, the host will no longer belong to that group. Each host knows which its current process belongs to. Because the relationship between group members is dynamic, the local multicast router to the local network will periodically poll the hosts in order to determine which hosts are still left in the group. If after a few polling, there are no longer members in a group, multi-wave router in the network will imagine that there are no hosts belonging to the group. The multicast router will not notice the status of group members to other multi-wave routers.

(4) the use of hardware multicast

Ethernet itself has hardware multicast capability. When the multicast packet is sent to the Ethernet, the Ethernet use hardware multicast to deliver to the host belonging to the group. Such a host may have multiple in the Ethernet.

Internet Number Authority IANA has assigned an Ethernet address block of the high-24bit for the 00-00-5E, that is, a number of Ethernet hardware address of the range is from 00-00-5E-00-00-00 to 00-00-5E-FF-FF-FF. Ethernet hardware address field 1 byte 1 is the lowest for the multicast address. As with one half of the IANA multicast address, that IANA has the Ethernet multicast address range is from 01-00-5E-00-00-00 到 01-00-5E-7F-FF-FF. Thus, only the 23bit are used for the Ethernet multicast address. This can only be a one to one relationship between Class D IP address and the 23bit. Class D IP addresses available for allocation are $28 \mathrm{bit}$. the first $5 \mathrm{bit}$ of the $28 \mathrm{bit}$ can not be used to constitute Ethernet hardware address.

\section{MUlti-Unit IN THE TANK AGAINST THE MULTICAST SIMULATION TRAINING SYSTEM}

A. The use of "hardware group approach" to achieve "the parent subnet multicast" design



Figure 5. Multicast grouping parent subnet

The design is based on the multicast application in LAN technology which uses hardware to realize the separation of a single tank. The basic idea is shown in Figure 5. We create a local area network by simulating three computers in each tank. They are connected to the HUB. One of the external relations of the drivers to configure a dual-card computer, in accordance with the proxy server software, use proxy server software to a second LAN card, as this small external link ports. The computer of the car drivers are connected with a multicast router. Bus drivers associated with the computer are set to the same multicast group to achieve multicast information exchange.

\section{B. The results of "parent subnet multicast"}

Ten simulated combat training tank in five to five group:

Adopting the network connection method before the "subnet multicast Father", there are 30 computers connected to the network. The information and messages of 30 computers sent and received are all in the network. The network utilization is $100 \%$ As for the driver computer, it needs not only interacting with a tank gunner and driving members with the station but also joint operations with the other 4 cars. Its information needed are $20 \%$ of all the required information. The gunner and driver for the corresponding computer just need interacting with the other two interactive computers. So the information they need is less than $7 \%$ of all information.

Through the above analysis, we can see that there are a lot of information redundancy in the original network and a large number of network resources are wasted.

After adopting the "Father of the subnet multicast" connection method, we can see that there are only 3 computers of a single sub-tank on the LAN internal exchanging information unhinderedly. Information exchange is no longer subject to the influence of other tanks and the information is very simple. There is no redundancy more than information. For operators, they need not judge the availability of information. All information is useful, which reduce the operational response time and improve the operations of the victory possible. For the father of multiple tanks in the LAN, the bus captains just send a message to a multicast group and the other drivers receive message directly by joining a multicast group. The connection method improve the real-time combat information and improve the operations of the victory greatly.

From the above analysis, the "Father of the subnet multicast" applications can indeed greatly improve efficiency of the combat simulation training system tank unit's and achieve the tank unit network optimization, simulation and training system.

\section{CONCLUSION}

Simulation training in joint operations is the inevitable trend of military training in the virtual battlefield environment. It is of inestimable significance to improve the quality, reduce equipment wear and tear, saving training funds. It will laid a strong technical base for building a largescale distribution Tactical Training System Synthesis for the future.

This paper analyzes design principles of network optimization and design process of a tank unit. It makes full use of the IP multicast technology and solves the problem of network data redundancy in large measures. 


\section{REFERENCES}

[1] THE ACADEMY, Zhang, Yang merit and so on. Distributed Interactive Simulation and military applications. Beijing: National Defence Industry Press, 2003.8.

[2] Jian-Guo Hao. High Level Architecture (HLA) in multiple federal interconnect technology and its implementation. Changsha: National Defense University, 2003.4
[3] Wang Chunzhong. Of Advanced Fire Control System Simulation, [MS Thesis]. Beijing: Armored Force Engineering Institute, 2002,3.

[4] Xie Xiren. Computer Networks (fourth edition), Electronic Industry Press, 2005.7 